\title{
Implementation of viscoelastic Hopkinson bars
}

\author{
R. Curry ${ }^{a}$, T. Cloete, and R. Govender \\ Blast Impact and Survivabillity Research Unit (BISRU), Department of Mechanical Engineering, \\ University of Cape Town, South Africa
}

\begin{abstract}
Knowledge of the properties of soft, viscoelastic materials at high strain rates are important in furthering our understanding of their role during blast or impact events. Testing these low impedance materials using a metallic split Hopkinson pressure bar setup results in poor signal to noise ratios due to impedance mismatching. These difficulties are overcome by using polymeric Hopkinson bars. Conventional Hopkinson bar analysis cannot be used on the polymeric bars due to the viscoelastic nature of the bar material. Implementing polymeric Hopkinson bars requires characterization of the viscoelastic properties of the material used. In this paper, $30 \mathrm{~mm}$ diameter Polymethyl Methacrylate bars are used as Hopkinson pressure bars. This testing technique is applied to polymeric foam called Divinycell H80 and H200. Although there is a large body of of literature containing compressive data, this rarely deals with strain rates above $250 \mathrm{~s}^{-1}$ which becomes increasingly important when looking at the design of composite structures where energy absorption during impact events is high on the list of priorities. Testing of polymeric foams at high strain rates allows for the development of better constitutive models.
\end{abstract}

\section{Introduction}

Material characterisation at high strain rates is concerned with measuring the change in mechanical properties, such as yield strength, work hardening, and ductility, at higher strain rates, which can deviate from quasistatic results $[1,2]$. The changes in these properties at high strain rates has become increasingly important as improvements have been made to the understanding of high speed machining operations, ballistic, and impact events where the changes in these properties can have adverse effects. Dynamic material characterisation forms the corner stone in developing accurate computational models. Mechanical properties are normally determined by performing standard loading tests such as compression, tension, or torsion $[3,4]$. Conventional testing can be used to achieve nominally constant loading rates for limited plastic strains and thereby a constant engineering strain rate. Quasi-static and low range dynamic tests are often performed on servo hydraulic or screw driven machines used to measure the stress-strain response of materials up to strain rates as high as $1 \mathrm{~s}^{-1}$. There are also purpose built machines that can achieve strain rates as high as $200 \mathrm{~s}^{-1}$. Typically if higher strain rates are desired impact driven stress wave propagation is used to load specimens. The main technique in the range of $500 \mathrm{~s}^{-1}-5000 \mathrm{~s}^{-1}$ is the Split-Hopkinson Pressure Bar (SHPB), which is capable of achieving high uniform uni-axial stress loading of a specimen in compression at nominally constant strain rates of the order of $10^{3} \mathrm{~s}^{-1}$. Hopkinson bar techniques have also been developed to probe the high-rate response of materials in tensile and torsion-loading stress states. In each instance, specimen stress is inferred using elastic elements in series with the specimen.

Typically stress waves are generated via an impact event, and the elastic elements used are long bars such that the duration of the loading pulse is less than the wave transit time in the bar. In Hopkinson bar techniques, the

a e-mail: richard.curry@uct.ac.za dynamic stress-strain response of materials at strain rates up to $5 \times 10^{3} \mathrm{~S}^{-1}$ in compression, and somewhat lower in tension or torsion, and true strains of 0.3 can be readily achieved in a single test. The most widely used techniques normally implement metallic Hopkinson bars [5], which can have a material impedance in the region of $40 \mathrm{MPa} s / \mathrm{m}$. These metallic bars experience difficulties when testing materials which have a significantly lower impedance than the bar being used to test them, meaning that testing of soft materials with an impedance of $2 \mathrm{MPa} s / \mathrm{m}$ is not possible. One solution to over coming this problem has been to simply use a bar material which has an impedance closer to that of the material being tested. As a result polymers have been used as Hopkinson bars as they have an impedance in the region of $4 \mathrm{MPa} \mathrm{s} / \mathrm{m}$. This paper deals with the implementation of polymeric Hopkinson bars which will enable testing of soft materials on a Split Hopkinson Pressure Bar (SHPB) setup.

\section{Characterising the viscoelastic effects}

The method chosen to characterise the viscoelastic wave propagation is Bacon's method [6]. This specific method was chosen due to the fact that the material is characterised experimentally in a straight forward way which lends itself to routine calibration. This streamlines the process with a calibration test being performed before each batch of testing, minimising any environmental deviation. In contrast, some of the other methods require at least one analytical component to be used, some requiring significant computational effort to derive a property that is assumed to remain constant. For instance changes in temperature have an effect on these properties, which is difficult to characterise. Bacon's method [6] describes viscoelastic material response with a complex modulus $E^{*}(\omega)$ which relates the stress and strainin the frequency domain at any point shown in the equation below.

$$
\tilde{\sigma}(x, \omega)=E^{*}(\omega) \tilde{\varepsilon}(x, \omega)
$$


The central feature of this method is the propagation coefficient $\gamma=\gamma(\omega)$ which is defined by

$$
\gamma^{2}(\omega)=-\frac{\rho \omega^{2}}{E^{*}(\omega)}
$$

The general solution to the one dimensional equation of motion becomes:

$$
\tilde{\varepsilon}(x, \omega)=\tilde{P}(\omega) e^{-\gamma x}+\tilde{N}(\omega) e^{\gamma x}
$$

where the functions $\tilde{P}(\omega)$ and $\tilde{N}(\omega)$ define the strains at $x=0$ of waves travelling in directions of increasing and decreasing $x$ respectively. From these it is possible to find the Fourier transform of the particle velocity $\tilde{v}(x, \omega)$ and the normal force $\tilde{F}(x, \omega)$ at any cross section $x$.

$$
\begin{array}{r}
\tilde{v}(x, \omega)=-\frac{i \omega}{\gamma}\left[\tilde{P}(\omega) e^{\gamma x}-\tilde{N}(\omega) e^{\gamma x}\right] \\
\tilde{F}(x, \omega)=-\frac{\rho A \omega^{2}}{\gamma^{2}}\left[\tilde{P}(\omega) e^{\gamma x}+\tilde{N}(\omega) e^{\gamma x}\right]
\end{array}
$$

The modulus and phase angle of the complex exponential functions $e^{-\gamma x}$ and $e^{\gamma x}$ are related to attenuation and propagation respectively. Thus the propagation coefficient $\gamma(\omega)$ is connected to the attenuation coefficient (or damping) $\alpha(\omega)$ and to the phase velocity $C(\omega)$ by

$$
\gamma(\omega)=\alpha(\omega)+i k(\omega)=\alpha(\omega)+i \frac{\omega}{C(\omega)}
$$

where $k(\omega)$ is the wave number defined by

$$
k=-\frac{\left(\Theta_{R}\right)-\left(\Theta_{I}\right)}{d}
$$

where $\Theta_{R}$ and $\Theta_{I}$ are the unwrapped phase angles of the two complex functions $\tilde{P}(\omega)$ and $\tilde{N}(\omega)$. So by finding $\alpha$ along with either $k$ or $C$, the propagation coefficient can be described.

The following properties of these different functions should be noted:

- The Wave number $k$ is an odd function

- Phase velocity $C$ is an even function

- The modulus and attenuation coefficient are even functions

In the work presented by Bacon [6] it is noted that the frequency range of importance while characterising polymeric materials is from $0-20 \mathrm{kHz}$. Above this point the frequency content becomes negligible as the high frequencies get damped out in the material. This results in excessive numerically induced noise as a result of division by very small numbers in the calculation of different components. For this reason the region that is highlighted in this work and presented in the following sections is from $0-20 \mathrm{kHz}$, although some content above this point is shown for completeness in the results.

\section{Experimental overview}

The experimental program consists of two processes, namely the characterisation of the polymeric bars and the material testing of the different polymeric foams. Emphasis is placed on the characterisation of the polymeric bars in order to lay a foundation for future work.

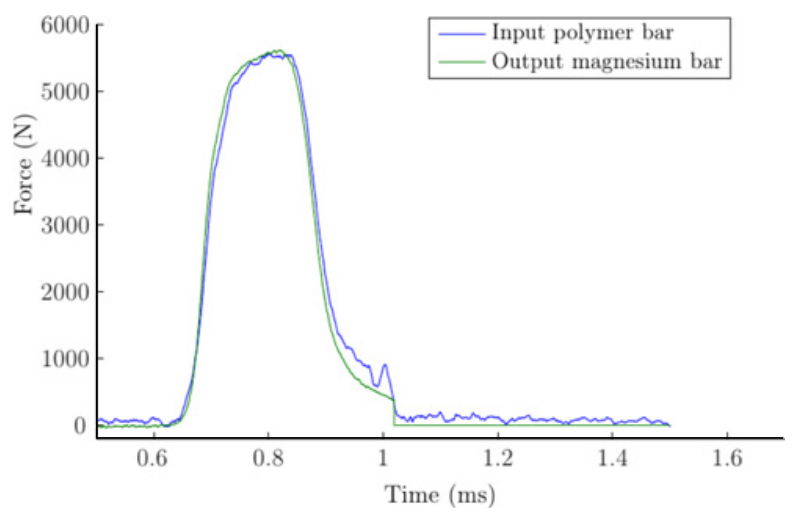

Fig. 1. Plot of the force balance between a Perspex input bar and a magnesium output bar.

\subsection{Perspex characterisation}

While Polycarbonate, Nylon and Perspex were intially considered as different bar material options, each was fully characterised and compared, ultimately Perspex bars were chosen due to the cost and accuray level to which stress waves could be characterised using Bacon's method [7]. The Attenuation co-efficient, wave number and phase velocity of the Perspex bar is shown in Figure 3. The figure represents a cloud of data captured over 15 discrete characterisation tests performed on the bars. The data is generally tightly grouped, forming a distinct trend up to $15 \mathrm{kHz}$. Between $15-20 \mathrm{kHz}$ the data cloud starts to disperse, and above $20 \mathrm{kHz}$ the bars have started to respond slightly differently depending on the inital impact with the striker. From this data a propagation co-efficient was selected that represents the trend and verified with a force balance at the bar ends using a magnesium bar.

In order to verify that the corrections made to the polymer bars are correct a magnesium bar was used to check the force balance at the interface. The results from the magnesium bar are trust worthy as they occour in a material which is well understood and known to be linear elastic below yield [8]. The results of the force balance at the interface of the two different bars are presented in Figure 1 and as can be seen the force at the bar ends matches verifying the polymer bar calibration.

\subsection{Deriving the propagation co-efficient}

The propagation coefficient $\gamma$ describes the changes that occur to a stress pulse that travels some distance through a viscoelastic material. When a stress pulse can be measured at two different positions in a Hopkinson bar, then the changes that occur to the pulse between these two points are assumed to be attributable to $\gamma$. There are two ways of achieving this;

- Using two gauge stations separated by some distance [9] on a Hopkinson bar and capturing the data from both. By comparing the two different signals the differences can be calculated.

- Using the reflection of a stress wave off a free end of the bar. This method requires taking the information 
from a single gauge station and separating the initial compressive pulse from the tensile reflection so that they can be compared in order to calculate $\gamma$.

In order to implement Bacon's method a set procedure needs to be followed. The first process is to window (i.e. separate) the incident and reflected pulses. Each window must contain all the pertinent information from the instant when the pulse starts to deviate from the mean zero to the time when the pulse settles back to the mean zero. Note that the full length of the signal is maintained and the data outside the area of interest is simply set to zero. From this point on the compressive incident pulse is a separate signal to the tensile reflection. Once each pulse has been separated and data set to zero where appropriate, a Fast Fourier Transform (FFT) function is used to transfer them to the frequency domain. The speed of this operation is optimised by ensuring the vector length is an exact power of two [7]. The shifting of the signals occours in the frequency domain utilising the propagation coefficient and an Inverse Fast Fourier Transform (IFFT) is used to tranfer the signal back to the time domain. Once this has been completed conventional Hopkinson bar analysis can be used to analyse the material response. Below the significant sections of the process are outlined for completeness.

\subsubsection{Magnitude}

The magnitude i.e. modulus of the complex number that results from the FFT is defined as:

$$
\text { modulus }=|F F T|
$$

where the right hand side is in polar notation. The Attenuation Ratio is therefore defined as:

$$
\text { Attenuation Ratio }=\frac{\mid \text { FFT Reflected pulse } \mid}{\mid \text { FFT Incident pulse } \mid}
$$

\subsubsection{Attenuation co-efficient}

The Attenuation coefficient $\alpha$ is defined as:

$$
\alpha=\frac{\ln \left(\frac{r_{R}}{r_{I}}\right)}{x}
$$

where $r_{R}$ and $r_{I}$ are the modulus of the reflected and incident pulses in the frequency domain respectively, while $x$ is the distance through which the pulse has propagated. Note that $\alpha$ is an even function in the frequency domain and defines the real part of the transfer function $\gamma$.

\subsubsection{Phase angle, wave number and phase velocity}

The phase angle oscillates between $\pi$ and $-\pi$, To make sense of this an operation called "unwrapping" needs to be performed on the phase angle to account for the jumps from $\pi$ to $-\pi$. This involves adding or subtracting $\pi$ depending on the jump that occurs. A noticeable difference can be seen as the phase angle changes between the

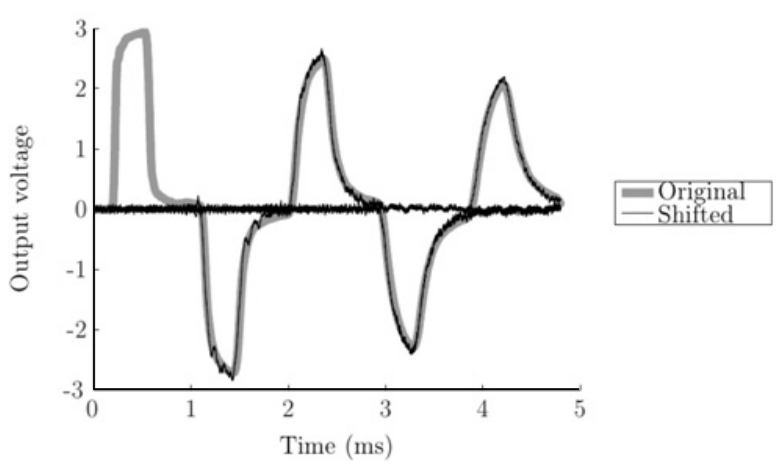

Fig. 2. Figure of the shifted signals overlaid with what they actually look like.

compressive and tensile pulses and a trend emerges. The wave number $k$ is defined as:

$$
k=-\frac{\left(\Theta_{R}\right)-\left(\Theta_{I}\right)}{x}
$$

where $\Theta_{R}-\Theta_{I}$ is the change in phase angle, and $x$ is the distance through which the pulse has propagated. Note that the wave number is an odd function in the freuency domain, and comprises the imaginary component of the transfer function $\gamma$. The Phase velocity can be derived from the wave number $k$ as follows:

$$
C(\omega)=\frac{\omega}{k(\omega)}
$$

where $\omega$ is the frequency. The phase velocity is an even function in the frequency domain.

\subsubsection{Propagation coefficient}

In order to shift an arbitrary pulse $\mathrm{P}$ along a bar the transfer function is multiplied by the distance $x$ of the shift and applied in the frequency domain as follows:

$$
P_{\text {shift }}=P e^{\gamma x}
$$

Once the shift has been applied then the pulse in the frequency domain, it is transferred to the time domain using the Inverse Fast Fourier Transform (IFFT).

In Figure 2 the incident pulse and the inverted tensile reflection have both been shifted to the free end of the bar where they should have the same magnitude as a result of the zero force boundary condition at the free surface. When shifting both these pulses in a bar to a common interface, one would expect them coincide. The fact that they do is regarded as confirmation that the method is working.

\subsubsection{Shifting stress waves in polymer bars}

The term "shifting" is used here to describe the process of taking a signal or stress wave captured at a gauge station on a Hopkinson bar, and predicting how that stress wave would appear if it had been captured at some position which is a distance $x$ from the gauge station. The stress wave of interest is separated or windowed from the data. After the stress wave has been windowed and the remainder of the data set to zero, the signal is transformed into the frequency domain by means of the FFT function.

$$
\varepsilon(t) \Rightarrow F F T \Rightarrow \tilde{\varepsilon}(\omega)
$$



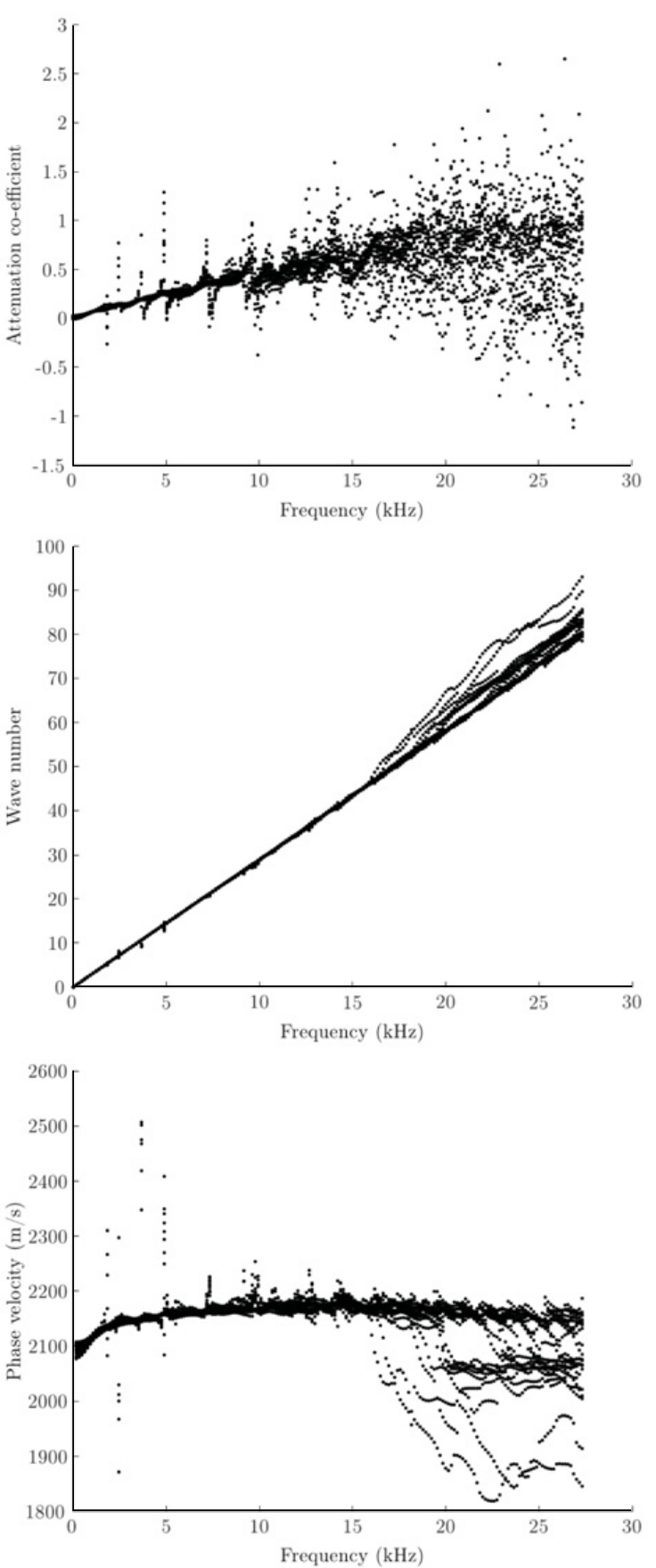

Fig. 3. Figure of the different properties of the Perspex bars selected for testing.

where $\tilde{\varepsilon}(\omega)$ is a frequency domain representation of the strain. At this point the propagation coefficient $\gamma(\omega)$ can be applied to "shift" the stress wave through a distance $x$ from the gauge station using the expression:

$$
\widetilde{\varepsilon}_{\text {shift }}(\omega)=\tilde{\varepsilon}(\omega) e^{\gamma x}
$$

In this form the shifted stress wave is expressed as a function of frequency and the inverse Fourier transform must be used to transfer the signal back to the time domain, where regular Hopkinson bar theory can be applied.

$$
\widetilde{\varepsilon}_{\text {shift }}(\omega) \Rightarrow I F F T \Rightarrow \varepsilon_{\text {shift }}(t)
$$

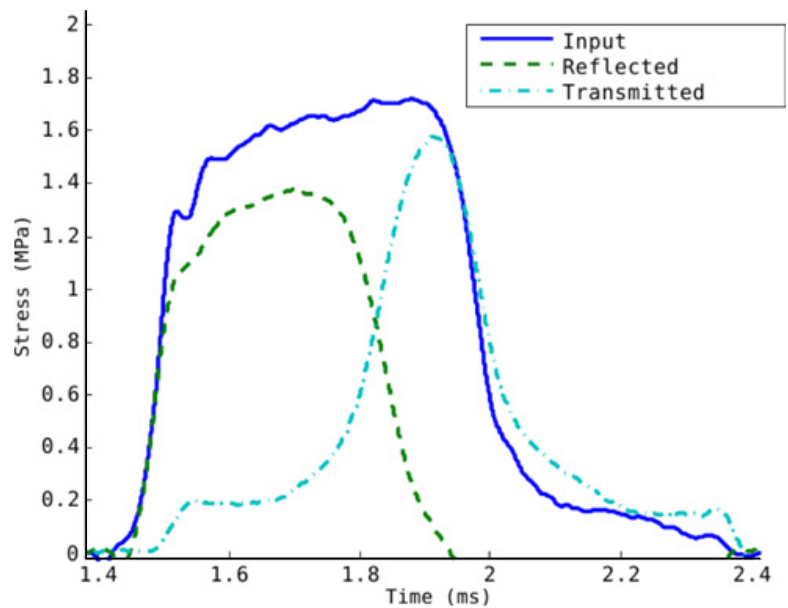

Fig. 4. Figure of signals captured at the strain gauge stations on the PMMA bars and shifted back to the material interface.

The result of a shift performed on PMMA bar signals using the propagation coefficient derived from the information in Figure 3 is shown in Figure 4. This result is typical of the results achieved using this method.

\subsection{Bar test equipment setup}

The Bars used were extruded circular Perspex bars, nominally $30 \mathrm{~mm}$ diameter. Both input and output bars were $2 \mathrm{~m}$ long and the gauge stations were placed in the center of each bar at $1 \mathrm{~m}$ from the bar end. This was done to ensure each bar could be characterised adequately. The strain gauge stations were set up using TML $350 \Omega$ strain gauges with a gauge length of $3 \mathrm{~mm}$ set up in a full bridge configuration with temperature compensation. The strain gauge amplifiers were designed and built in house and set with a gain of 1000 and independant bridge voltages of $\pm 1.4 \mathrm{~V}$ for the input bar and $\pm 2.4 \mathrm{~V}$ for the output bar. Each bar and gauge station was then calibrated and verified using the magnesium bar to ensure the results were accurate. A polycarbonate striker $20 \mathrm{~mm}$ in diameter and $400 \mathrm{~mm}$ long was used for calibration.

\subsection{Specimen preparation}

Two different densities of foam were tested, namely Divinycell H80 and H200. Quasistatic and dynamic tests were performed on both. The specimens were cored from sheets of the foam using a hole saw leaving cylindrical specimens of $28 \mathrm{~mm}$ diameter and $25 \mathrm{~mm}$ long for the H80 foam and $14 \mathrm{~mm}$ diameter and $14 \mathrm{~mm}$ long for the $\mathrm{H} 200$ foam which were easily mounted between the bars. The different dimentions were selected to ensure the specimens equlibrated appropriately. Figure 5 shows the typical specimen equilibration achieved using this method.

Care had to be taken to ensure the feed rate was adequate for the density of foam being cut to ensure the cutter did not rip or tear the material. Each specimen was measured to ensure the geometry was regular and very little variance was found using this method. 


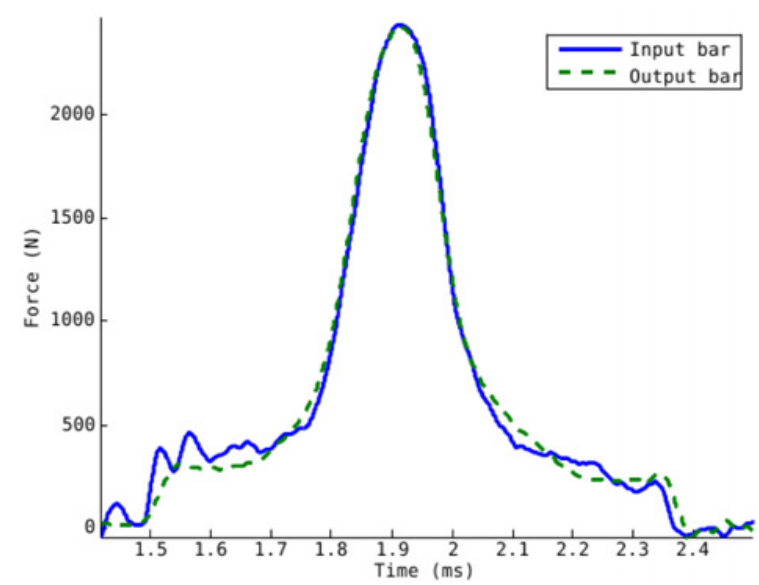

Fig. 5. Figure showing the force on the two ends of a typical specimen.

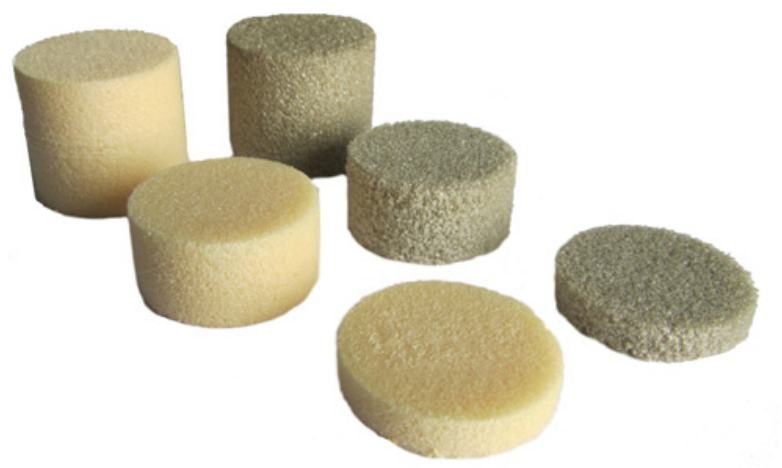

Fig. 6. The foam specimens to be tested.

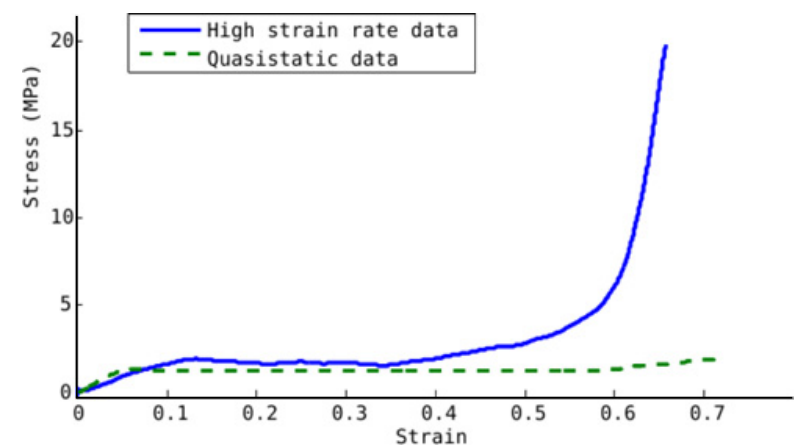

Fig. 7. Figure showing the typical Stress $v s$. Strain results of the H80 specimens.

\section{Results and discussion}

The quasistatic tests were performed at strain rates of $1.3 \times 10^{-2} \mathrm{~s}^{-1}$ and $10^{-1} \mathrm{~s}^{-1}$, while the strain rate of the dynamic tests covered a range between $400-2400 \mathrm{~s}^{-1}$.

Figures 7 and 8 show the typical Stress vs. Strain response for each material in both quasistatic and high strain rate testing. Each material clearly exhibits the classic linear elastic, plateau and densification phases. All these phases are discernable in the data of both the quasistatic and dynamic tests. This data is very similar to that obtained in the literature by Tagarielli et al [10]. The strain rates achieved in the dynamic tests had an almost constant initial

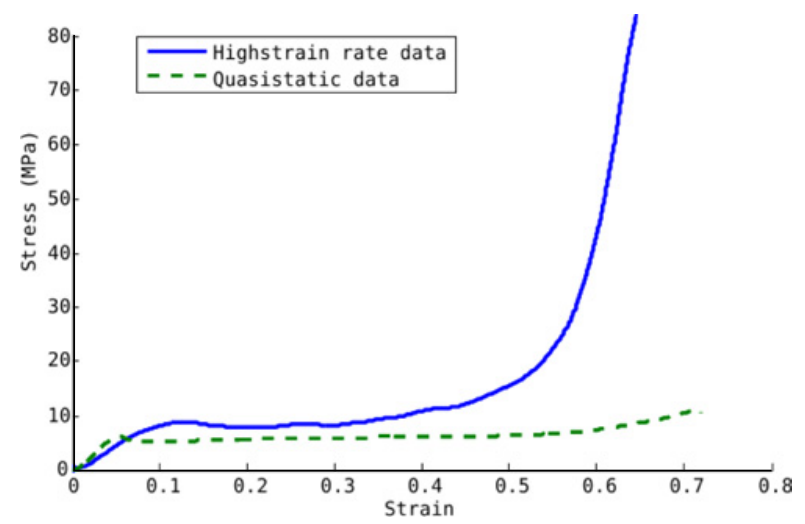

Fig. 8. Figure showing the typical Stress $v s$. Strain results of the H200 specimens.

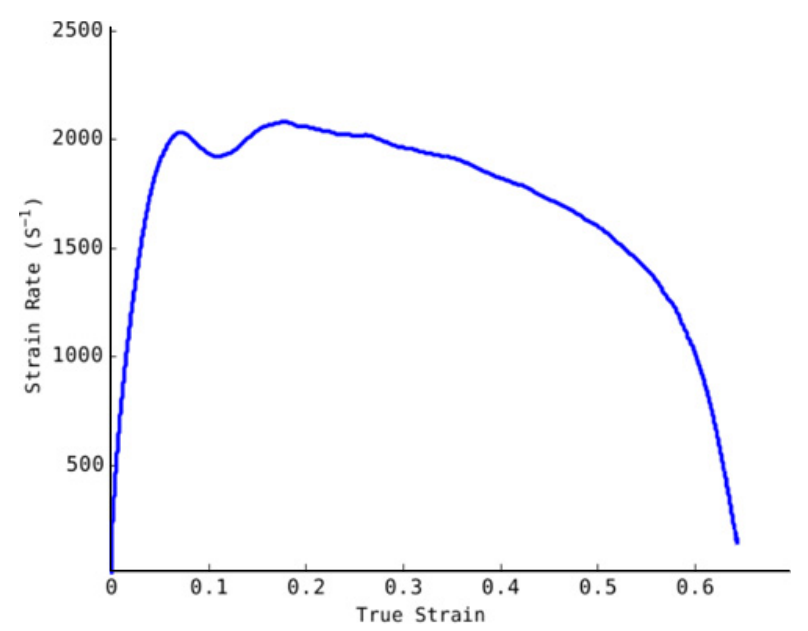

Fig. 9. Figure showing the typical Strain rate $v s$. Strain results of the specimens.

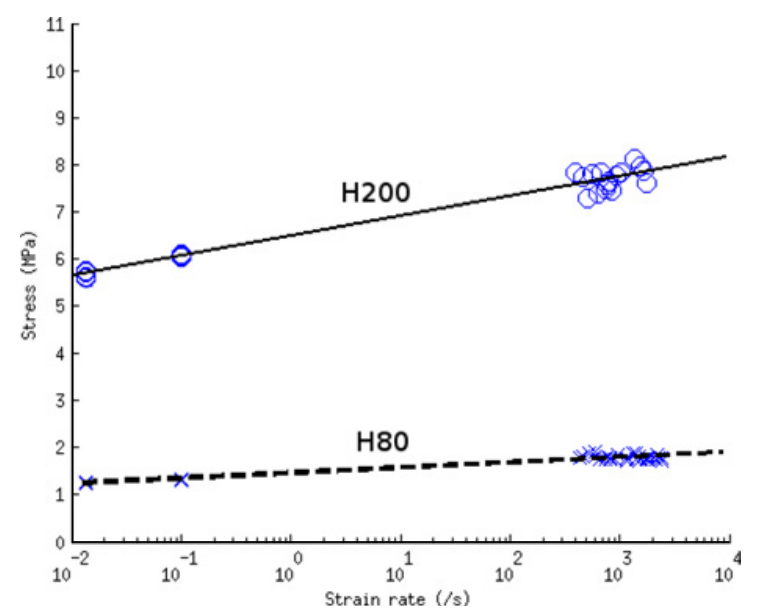

Fig. 10. Figure showing the results of the rate dependence of $\mathrm{H} 80$ and $\mathrm{H} 200$ measured at $15 \%$ strain.

section followed by a gradual decline back to zero as seen in Figure 9.

The stress at a strain of $15 \%$ is compared for different strain rates in Figure 10 and a clear trend can be seen. H80 shows a very gradual rate sensitivity while H200 hasa slightly more pronounced sensitivity. This is a similar response to that reported by Ouellet et al [11] who 
tested other polymeric foams up to strain rates of around $1000 \mathrm{~s}^{-1}$.

\section{Conclusion}

In this paper, a viscoelastic split Hopkinson bar setup which can be used for the testing of materials with low impedance has been implemented. Bacons' method was chosen and implemented to deal with the viscoelastic effects of the bar material and the method has been implemented computationally and validated through force balance tests using magnesium bars together with the polymer bars. Divinycell H80 and H200 foams were tested using the method outlined and the results achieved correlate with similar materials found in the literature. Further testing of different polymeric foams and other soft materials can now be conducted.

\section{Acknowledgements}

Special thanks go to the UCT mechanical engineering Department and staff of BISRU for the help and support provided. A special thanks to Ass-Prof Genevieve Langdon for the polymeric foam material supplied.

\section{References}

1. R. Bouix, P. Viot, J. lataillade, Int jnl impact engineering, 36 (2009) 329-342

2. S. Kiernan, L. Cui, M. D. Gilchrist, Int jnl non-lin mech, 44 (2009) 456-468

3. ASTM E8/E8M - 09 Standard Test Methods for Tension Testing of Metallic Materials (2010)

4. ASTM E9-09 Standard Test Methods of Compression Testing of Metallic Materials at Room Temperature (2010)

5. G. T. Gray(III), ASM Handbook, Volume 08 Mechanical Testing and Evaluation (2000)

6. C. Bacon, Exp mech, 38 (1998) 242-249

7. R. Curry, MSc Diss, Implementation of viscoelastic hopkinson bars, University of Cape Town (2010)

8. R. M. Davies, Phil trans royal society A 240 (1948) 375-457

9. D. T. Casem, W. Fourney, P. Chang, Ploymer testing 22 (2003) 155-164

10. V. Tagarielli, V. Deshpande, N. Fleck, Composites: Part B 39 (2008) 83-91

11. S. Ouellet, M. Worswick, D. Cronin, Polymer testing 25 (2006) 731-743 